\title{
Down syndrome and moyamoya: clinical presentation and surgical management
}

\author{
Alfred P. See, MD, ${ }^{1}$ Alexander E. Ropper, MD, ${ }^{1}$ Daniel L. Underberg, BA, ${ }^{1}$ \\ Richard L. Robertson, MD, ${ }^{2}$ R. Michael Scott, MD, ${ }^{1}$ and Edward R. Smith, MD ${ }^{1}$ \\ Departments of ${ }^{1}$ Neurological Surgery and ${ }^{2}$ Radiology, Boston Children's Hospital and Harvard Medical School, Boston, \\ Massachusetts
}

\begin{abstract}
OBJECT Moyamoya can cause cerebral ischemia and stroke in Down syndrome (DS) patients. In this study, the authors defined a surgically treated population of patients with DS and moyamoya and compared their clinical presentation, response to surgical treatment, and long-term prognosis with those of the general population of patients with moyamoya but without DS.
\end{abstract}

METHODS This study was a retrospective review of a consecutive operative series of moyamoya patients with DS treated at Boston Children's Hospital from 1985 through 2012.

RESULTS Thirty-two patients, average age 9.7 years (range 1.8-29.3 years), underwent surgery for moyamoya in association with DS. The majority presented with ischemic symptoms ( $87 \%$ stroke, $42 \%$ transient ischemic attacks). Twentyfour patients (75\%) had congenital heart disease. Nineteen patients (59\%) had bilateral moyamoya on presentation, and 13 presented with unilateral disease, of which 2 progressed to surgery on the opposite side at a later date. Patients were followed for a median of 7.5 years (1-20.2 years) after surgery, with no patients lost to follow-up. Follow-up arteriography demonstrated Matsushima Grade A collaterals in 29 of 39 (74\%) hemispheres, Grade B in $5(13 \%)$, and Grade C in 5 $(13 \%)$.

Complications included postoperative strokes in 2 patients, which occurred within 48 hours of surgery in both; one of these patients had arm weakness and the other confusion (both had recovered completely at follow-up). Seizures occurred in 5 patients perioperatively, including one who had a new seizure disorder related to hypocalcemia.

CONCLUSIONS Moyamoya disease is a cause of stroke in patients with DS. Both the incidence of preoperative stroke ( $87 \%$ vs $67 \%$ ) and the average age at diagnosis for children under age 21 (8.4 vs 6.5 years) were greater in patients with DS and moyamoya than in the general moyamoya surgical population, suggesting a possible delay in reaching a correct diagnosis of the cause of cerebral ischemia in the DS patient population. Pial synangiosis provided long-term protection from stroke in all patients treated.

http://thejns.org/doi/abs/10.3171/2014.12.PEDS14563

KEY WORDS moyamoya; Down syndrome; pial synangiosis; stroke; revascularization; vascular disorders

$\mathrm{M}$ OYAMOYA is a progressive arteriopathy characterized by narrowing of the internal carotid arteries, and the vessels of the circle of Willis, leading to compensatory collateral vessel development and risk of stroke..$^{13}$ Initially described as an idiopathic process, there is now an increasing frequency of isolated case reports attesting to the improved recognition and diagnosis of moy- amoya associated with various genetic and systemic disorders. However, large series focused on detailed study of specific populations known to be at risk of moyamoya remain limited. Here we present our institutional experience reviewing the clinical presentation, radiographic findings, and surgical treatment of a series of 32 patients with Down syndrome (DS) and moyamoya.

ABBREVIATIONS DS = Down syndrome; DSA = digital subtraction angiography; MRA = MR angiography; mRS = modified Rankin Scale; TIA = transient ischemic attack. SUBMITTED October 14, 2014. ACCEPTED December 15, 2014.

INCLUDE WHEN CITING Published online April 3, 2015; DOI: 10.3171/2014.12.PEDS14563.

DISCLOSURE The authors report no conflict of interest concerning the materials or methods used in this study or the findings specified in this paper. 


\section{Methods}

A consecutive surgical series of patients with moyamoya arteriopathy was reviewed to identify all patients with both moyamoya and DS who were treated with surgical revascularization between 1985 and 2012. Moyamoya was confirmed by the criteria outlined in the Japanese guidelines and recent pediatric evidence-based practice review. ${ }^{4,16}$

In accordance with an institutional review board-approved protocol, medical records were retrospectively reviewed to determine patient demographic information, age at presentation and initial surgery, symptoms at presentation, comorbid conditions, results of radiographic studies, perioperative and late complications, and long-term outcomes. Modified Rankin Scale (mRS) scores obtained at late follow-up and Matsushima grade on postoperative digital subtraction angiography (DSA) were used to evaluate the outcome of this cohort. ${ }^{17,18,20}$

\section{Results}

\section{Demographic Characteristics}

A total of 500 patients underwent surgery for moyamoya during the study period. Thirty-two $(6.4 \%$ of the total series) of those patients had both moyamoya and DS and were included in this study.

Demographic information of the study cohort was summarized in Table 1 . There were 15 females and 17 males in the group. The average age at time of treatment was 9.7 years (range 1.8-29.3 years). Twenty-nine of the 32 patients were 21 years of age or younger at the time of

TABLE 1. Demographic characteristics of the patients included in this study

\begin{tabular}{|c|c|}
\hline Characteristic & Value \\
\hline No. of patients & $32^{*}$ \\
\hline \multicolumn{2}{|l|}{ Sex } \\
\hline Female & $15(47 \%)$ \\
\hline Male & $17(53 \%)$ \\
\hline \multicolumn{2}{|l|}{ Age at surgery (yrs) } \\
\hline Mean & 9.7 \\
\hline Range & $1.8-29.3$ \\
\hline \multicolumn{2}{|l|}{ Pre-existing medical conditions } \\
\hline $\begin{array}{l}\text { Structural cardiac disease (tetralogy of Fallot, ven- } \\
\text { tricular septal defects, etc.) }\end{array}$ & $24 / 32(75 \%)$ \\
\hline Endocrine disorder & $14(44 \%)$ \\
\hline Hypothyroid & 10 \\
\hline Hyperthyroid & 3 \\
\hline Diabetes mellitus & 1 \\
\hline $\begin{array}{l}\text { Gastrointestinal disorder (reflux, Meckel's diverticu- } \\
\text { lum, Hirschprung's disease, duodenal atresia) }\end{array}$ & $12(38 \%)$ \\
\hline Severe developmental delay & $10(19 \%)$ \\
\hline Seizure disorder & $2(6 \%)$ \\
\hline Leukemia & $2(6 \%)$ \\
\hline Shunt-treated hydrocephalus & $1(3 \%)$ \\
\hline
\end{tabular}

* Represents $6.4 \%$ of the 500 patients who underwent surgery (816 surgical cases) for moyamoya during the study period (June 1985-November 2013). treatment $(\mathrm{n}=29$, average age 8.4 years). Preexisting medical conditions included structural cardiac disease in 24 patients $(75 \%)$, endocrine disorders in 14 patients $(44 \%)$, gastrointestinal disorders in 12 patients (38\%), and other conditions outlined in Table 1 (some individuals had more than 1 condition, making the total number of conditions greater than 32).

\section{Clinical Presentation}

The average age at diagnosis was 9.5 years (range 1.6-29.1 years) and all patients had symptoms referable to moyamoya at the time of presentation (100\%) (Table 2). A stroke or fixed deficit was present in 27 patients (87\%), transient ischemic attacks (TIAs) were documented in 14 patients (42\%), seizures were present in 8 patients (26\%), and 1 patient presented with intracranial hemorrhage.

\section{Radiographic Presentation}

There were 51 affected hemispheres in 32 patients $(25$ left, 26 right) (Table 3). Bilateral disease was present in 19 patients (59\%) at presentation and 8 individuals (25\%) had posterior circulation involvement. Thirty-one patients (97\%) had radiographic evidence of infarction on imaging prior to surgery, and the median Suzuki stage was 4 for involved hemispheres (Fig. 1).

\section{Surgical Results}

A total of 51 operations were performed in 32 patients (Table 4). Thirteen patients (32\%) underwent unilateral surgery, and 19 patients (68\%) underwent bilateral procedures. Pial synangiosis was the primary operative technique in $49(96 \%)$ of the 51 cases; this technique has been described in detail in previous publications. ${ }^{13,14}$ Direct superficial temporal to middle cerebral artery (STA-MCA) bypass was performed in 2 adult cases and was chosen because of availability of large donor and recipient vessels coupled with what was perceived to be crescendo-type presentation of symptoms. The average length of stay was 6 days, including the planned admission the day before surgery for intravenous hydration (part of our institutional protocol).

\section{Complications}

In the 51 operations, there were no deaths. Complica-

TABLE 2. Clinical presentation in 32 patients

\begin{tabular}{lc}
\hline \multicolumn{1}{c}{ Characteristic } & Value \\
\hline Age at diagnosis (yrs) & \\
\hline Mean & 9.5 \\
\hline Range & $1.6-29.1$ \\
\hline Symptomatic at presentation & $32(100 \%)$ \\
\hline Clinical findings & $27(87 \%)$ \\
\hline Stroke/fixed deficit & $14(42 \%)$ \\
\hline TIA & $8(26 \%)$ \\
\hline Seizure & $1(3 \%)$ \\
\hline Hemorrhage (post-XRT for ALL)
\end{tabular}

$\mathrm{ALL}=$ acute lymphocytic leukemia; $\mathrm{XRT}=$ radiation therapy. 
TABLE 3. Radiographic presentation in 32 patients

\begin{tabular}{lc}
\hline \multicolumn{1}{c}{ Variable } & Value \\
\hline Total no. of affected hemispheres & $51(25 \mathrm{It} / 26 \mathrm{rt})$ \\
\hline Bilateral disease & $19 / 32(59 \%)$ \\
\hline Unilateral disease & $13 / 32(41 \%)$ \\
\hline Lt hemisphere & $6 / 13(46 \%)$ \\
\hline Rt hemisphere & $7 / 13(54 \%)$ \\
\hline Posterior circulation involvement & $8 / 32(25 \%)$ \\
\hline Radiographic stroke & $31 / 32(97 \%)$ \\
\hline Hemorrhage & $1 / 32(3 \%)$ \\
\hline Suzuki stage & 4 \\
\hline Median & $1-5$ \\
\hline Range
\end{tabular}

tions included postoperative strokes in 2 patients, occurring within 48 hours of surgery in both cases; 1 of these patients showed arm weakness and the other confusion (both patients had recovered completely at follow-up) (Table 5). One patient had a perioperative TIA with a negative MRI (1.9\%). Seizures occurred in 5 patients perioperatively $(9.8 \%), 2$ patients had infections requiring antibiotic treatment without reoperation $(3.9 \%)$, and 2 patients developed delayed subdural hematomas requiring operative drainage $(3.9 \%)$ at 48 and 54 days postoperatively.

\section{Outcomes}

Follow-up was available for all patients, with an average duration of 7.5 years (range 1-20.2 years) (Table 6). After

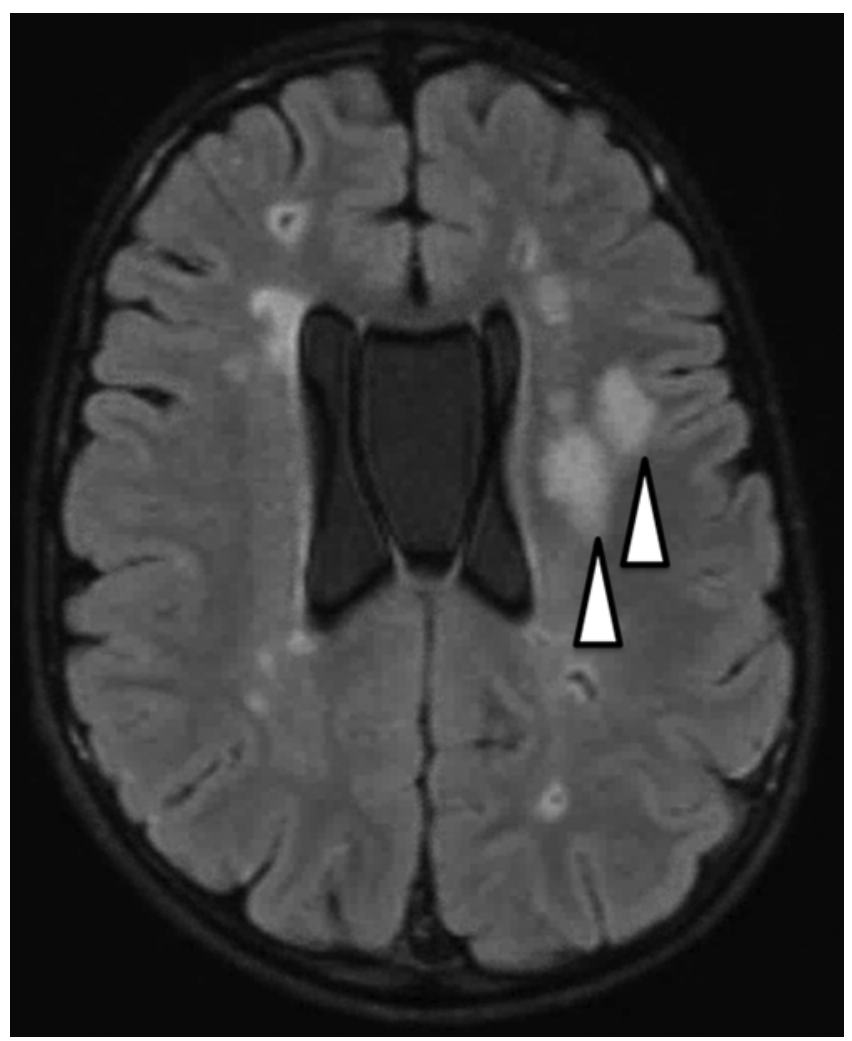

FIG. 1. Axial FLAIR MR image demonstrating numerous infarcts in a child with DS (larger infarcts indicated by arrowheads).
TABLE 4. Surgical results

\begin{tabular}{cc}
\hline \multicolumn{1}{c}{ Variable } & Value \\
\hline No. of operations & 51, in 32 patients \\
\hline Unilateral & $13 / 51(32 \%)$, in 13 patients \\
\hline Bilateral & $38 / 51(68 \%)$, in 19 patients \\
\hline Type of operation & \\
\hline Pial synangiosis & $49 / 51(96 \%)$ \\
\hline Direct STA-MCA bypass & $2 / 51(4 \%)$ \\
\hline Length of stay (no. of days) & \\
\hline Average & 6 \\
\hline Range & $4-14$ \\
\hline
\end{tabular}

STA-MCA = superficial temporal artery to middle cerebral artery.

* Includes 1 day of preoperative hydration.

discharge (excluding the perioperative complications previously noted in Table 5), there were no deaths, no clinical strokes, 3 TIAs (6\%/7.5 years), 5 new seizures (10\% per hemisphere, $16 \%$ per patient, with 1 patient developing a long-term seizure disorder related to hypocalcemia, a $2 \%$ risk of long-term seizure disorder), and 3 patients with headaches lasting more than 3 months (9.4\%).

Clinical status was stable or improved in 31 patients (97\%) according to comparison of preoperative modified Rankin Scale (mRS) scores to postoperative mRS scores. One patient (3\%) was clinically worse, because of a new seizure disorder secondary to chronic hypocalcemia. In this patient, repeated postoperative imaging studies (including after the onset of the seizure disorder) never revealed any new stroke or obvious cause related to moyamoya or surgical complications.

In 31 patients (97\%) imaging studies were performed at 1 -year (or subsequent) follow-up visits. Moyamoya arteriopathy progression was documented on MR angiography (MRA) in 25 (81\%) of 31 patients, yet no patients ( 0 of 31 ) had evidence of new strokes on imaging studies obtained 1 year or more after hospital discharge. In addition to this radiographic evidence of stroke prevention, postoperative imaging also corroborated the success of surgical revascularization, with new surgical collaterals and/or patent bypass being evident in $50(100 \%)$ of 50 hemispheres (as determined independently by radiologists reading postoperative films as part of clinical practice and defined as new, transdural vessels visualized within the area of the surgical field). While we recommended postoperative DSA to all patients, some parents/patients declined or were unable to travel for the procedure. Angiographic data with Mat-

TABLE 5. Complications

\begin{tabular}{|c|c|}
\hline Complication & No. of Cases (\%) \\
\hline Death & $0(0 \%)$ \\
\hline Stroke & $2 / 51(5.9 \%)$ \\
\hline TIA & $1 / 51(1.9 \%)$ \\
\hline Seizure & $5 / 51(9.8 \%)$ \\
\hline Infection & $2 / 51(3.9 \%)$ \\
\hline Subdural hematoma requiring operation & $2 / 51(3.9 \%)^{*}$ \\
\hline
\end{tabular}

* At 48 and 54 days after surgery. 
TABLE 6. Outcomes

\begin{tabular}{lc}
\hline \multicolumn{1}{c}{ Variable } & Value \\
\hline Duration of follow-up (yrs) & \\
\hline Mean & 7.5 \\
\hline Range & $1-20.2$ \\
\hline Clinical outcomes (following discharge) & $0(0 \%)$ \\
\hline Death & $0 / 51(0 / \%)$ \\
\hline Stroke & $3 / 51(6 \%)$ \\
\hline TIA & $5 / 51(10 \%) ; 5 / 32$ patients \\
\hline Seizure & $(16 \%)$ \\
\hline Headache & $3 / 51(5.8 \%) ; 3 / 32$ patients \\
& $(9.4 \%)$ \\
\hline Clinical status (1 yr postop) & $31 / 32(97 \%)$ \\
\hline Stable or improved & $1 / 32(3 \%)$ \\
\hline Worsened & \\
\hline Radiographic outcomes $\dagger$ & $21 / 32(66 \%)$ \\
\hline MRI/MRA only & $10 / 32(31 \%)$ \\
\hline Angiography (w/ or w/o MRI) & $1 / 32(3 \%)$ \\
\hline No imaging & $25 / 31(81 \%)$ \\
\hline Moyamoya progression (1 yr postop) & $5 / 39(13 \%)$ \\
\hline New strokes (after discharge) & patients) \\
\hline Surgical collaterals/patent bypass & $50 / 50(100 \%)$ hemispheres \\
\hline Matsushima grades $\ddagger$ & \\
\hline A & $29 / 39(74 \%)$ \\
\hline B & $5 / 39(13 \%)$ \\
\hline C & \\
\hline
\end{tabular}

* New seizure disorder $w /$ chronic hypocalcemia, no stroke.

† A total of $31(97 \%)$ of the 32 patients had follow-up imaging studies obtained at least 1 year after surgery.

$\ddagger$ Available for 39 hemispheres.

sushima grades were available for 39 of the 51 operated hemispheres. Of these, 29 (74\%) had Grade A collaterals, 5 (13\%) Grade B, and 5 (13\%) Grade C (Fig. 2).

\section{Discussion}

Moyamoya occurs with a higher frequency in DS than in the general pediatric population, with recent reports suggesting a 26-fold difference in moyamoya prevalence between children with and without DS. ${ }^{9}$ The estimated prevalence of DS was 3.8\% among all patients admitted with moyamoya disease and $9.5 \%$ among patients with moyamoya who were younger than 15 years, findings that are generally concordant with high-volume center experience, including results published from our institution. ${ }^{7,9,11,12}$ Yet, despite this association-predicting thousands of affected individuals worldwide-there have been fewer than 80 cases of moyamoya syndrome in association with DS reported in the literature, with the single largest series, now nearly a decade old, encompassing 16 patients (PubMed search, September 10, 2014). ${ }^{1,37-9,11}$ This dated and limited experience underscores the need to provide physicians who treat patients with DS and individuals with moyamoya with current, critical data, such as presenting symptoms, radiographic findings, and response to surgical treatment.

This study represents the largest series to date of DS patients with moyamoya surgically treated with cerebral revascularization. In addition to the important objectives of updating the literature with markedly increased numbers of patients (32 vs 16) and length of follow-up (mean 7.5 vs 5.6 years), the primary goals of this research include: 1 ) defining features of the moyamoya syndrome associated with DS (are they different from non-DS moyamoya?) and 2) determining the results of surgical revascularization in these patients at early and late follow-up (does treatment work?).

Features of non-DS-associated moyamoya have been published previously, including series from our own institution. Data from our same team using identical protocols for both DS and non-DS moyamoya facilitates a direct comparison between the 2 patient populations. We believe that these data help to characterize unique aspects of DSrelated moyamoya (DS moyamoya) and will improve the delivery of care to this particular patient population and identify areas of future study.

\section{Unique Features of DS-Related Moyamoya}

Patients with DS and moyamoya were diagnosed in this patient population at an older age than those with non-DS moyamoya (comparing identical groups of only pediatric patients, 21 years of age or younger), with age at presentation being 8.4 years for the DS group versus 6.5 years for the group without DS, and with a higher rate of symptomatic presentation $-100 \%$ versus $\sim 75 \%$ in non-DS moyamoya. All DS patients in this series presented with some ischemic symptoms, with higher rates of completed strokes $-87 \%$ of patients with DS moyamoya had strokes versus $68 \%$ of those with non-DS moyamoya. In addition, DS-moyamoya patients more commonly had seizures prior to diagnosis, in contrast to those with non-DS moyamoya $-26 \%$ versus $6 \%$.

This increase in clinical findings was concordant with the radiographic data, in which nearly all DS-moyamoya patients $(97 \%)$ had radiographic evidence of stroke prior to surgery (vs $\sim 50 \%$ of the patients with non-DS moyamoya), higher Suzuki stages (median of 4 vs average of 3.1) in involved hemispheres, and a greater likelihood of involvement of the posterior circulation $(25 \%$ vs $<5 \%)$. However, there were no unique anatomical or radiographic characteristics that individually distinguished DS-related moyamoya from non-DS moyamoya. Rather, the aggregate of these data suggest that patients with DS have more advanced-but otherwise similar-disease at presentation compared with non-DS moyamoya counterparts.

Given the unique group of medical conditions linked with DS-including cardiac disease, endocrine disorders, severe developmental delay, and gastrointestinal disease (see Table 1)-it is tempting to speculate that some component of the more advanced arteriopathy identified in this study may be attributed, in part, to obfuscation of diagnosis. Developmental delay may hinder communication about new symptoms, and many of the symptoms of moyamoya can be mimicked by the other conditions noted 


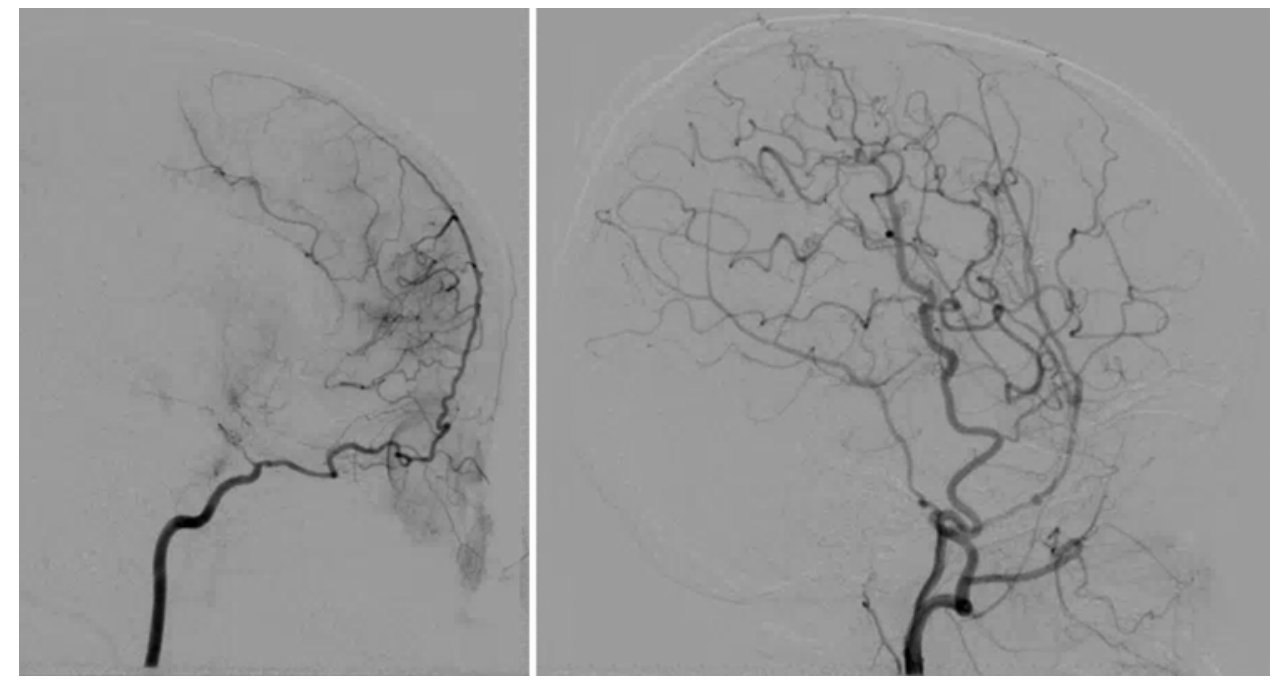

FIG. 2. Lateral projections of digital subtraction angiogram obtained in a DS patient demonstrating advanced Suzuki stage on selective left internal carotid artery injection (left) and robust, Matsushima Grade A collaterals following pial synangiosis on selective lateral left external carotid artery injection (right).

in Table 1. Moreover, even after a stroke is diagnosed, it can potentially be incorrectly ascribed to an embolic event from cardiac disease, delaying identification of the intracranial arteriopathy. Consequently, one important conclusion from this study is that recognition of moyamoya as a major stroke risk in DS patients may expedite its diagnosis and reduce the burden of structural brain injury prior to treatment. We propose that primary care providers should consider the possibility of moyamoya as a cause of stroke in DS patients when new neurological deficits are detected (even if transient) and suggest that noninvasive imaging with MRI/MRA would be prudent, both to determine if a stroke has occurred and to assess for the presence of moyamoya. If an arteriopathy is detected, referral to a pediatric neurosurgeon experienced with DS moyamoya may be warranted. Making definitive recommendations regarding general MRI/MRA screening of children with DS is beyond the scope of this paper, but it is our hope that the results reported here may prompt a broader discussion by relevant caregivers.

\section{Efficacy of Surgical Revascularization in DS Patients With Moyamoya}

Patients with DS and moyamoya had higher rates of complications with surgical treatment compared with non-DS moyamoya patients. The perioperative stroke rate per hemisphere was $5.9 \%$ (vs 4\%) and-in particular-the risk of seizure was high at $16 \%$ per patient for seizure at some point during the follow-up period, with an immediate perioperative seizure risk of $\sim 10 \%$ /hemisphere. ${ }^{12-14,19}$ These results reinforce the hypothesis that clinical status at the time of surgery directly relates to surgical outcome and argue that earlier diagnosis-when the disease is presumably less severe-may reduce the risk of complications. ${ }^{12-14}$ Specific to DS, coordinating care with relevant medical subspecialties (such as cardiology, endocrinology, gastroenterology, etc.) preoperatively may be important in reducing anesthetic and perioperative risks.

Interestingly, once treated, patients with DS-related moyamoya have robust and durable responses to revascularization, both clinically and radiographically. Clinically, this series of patients demonstrated stability or improvement in their mRS scores in $97 \%$ of cases, which is equivalent or better than in most reported series including non-DS moyamoya patients. . $^{5,13,14,19}$ Remarkably, the radiographic findings were even better than the excellent results of the non-DS group, with no DS moyamoya patients demonstrating new strokes after hospital discharge, despite progressive arteriopathy in the circle of Willis in $81 \%$ of individuals. Surgical collaterals were documented in $100 \%$ of cases, and the percentage of patients with the best Matsushima grade was higher in DS patients than in the non-DS moyamoya population (74\% vs $65 \%$ for Grade A). These findings were durable, with protection from stroke evident over an average of 7.5 years of follow-up. Taken together, these results suggest that DS-moyamoya patients may be higher risk surgical candidates but derive a greater long-term benefit from treatment once revascularized.

\section{Potential Limitations of this Study and Recommendations From the Findings}

There are several limitations to be considered in interpreting these results. The study was a retrospective case series, and the sample size was small. Postoperative angiography was declined or unavailable in some cases, which could introduce additional selection bias. Nevertheless, some results are clear. Our findings indicate that DS patients with moyamoya present at older ages and are more likely to have seizures and completed strokes than patients with moyamoya without DS, suggesting a delay in diagnosis. Radiographically, DS-associated moyamoya is largely indistinguishable from non-DS moyamoya, although DS-moyamoya patients have higher radiographic infarction rates, posterior circulation involvement, and Suzuki stages. DS-moyamoya patients are medically complex and have higher perioperative complication rates, but they demonstrate excellent long-term clinical and ra- 
diographic outcomes. All patients in our study remained clinically and radiographically stroke free on long-term follow-up, despite evidence of angiographic progression of moyamoya in $81 \%$ of operated hemispheres. Consequently, we conclude that revascularization surgery in these patients seemed to be the major factor in preventing cerebral infarcts in the face of worsening stenosis of the cerebral arteries.

\section{Conclusions}

Moyamoya disease is an important cause of stroke in patients with DS. The results of this study support the hypothesis that the arteriopathy of moyamoya is diagnosed at a later time, with more advanced progression of disease, in patients with DS than in moyamoya patients without DS. Surgical revascularization has slightly higher risks in DS patients but ultimately confers favorable outcomes and long-lasting protection from stroke. Taken together, our results underscore the potential merit of 2 future initiatives. First, greater awareness of the association of DS and moyamoya is needed among allied health care providers who treat patients with DS. It is reasonable to infer that better knowledge of an at-risk population would aid in earlier diagnosis and treatment. Second, the potential for screening DS patients for moyamoya should be considered. Success in screening at-risk populations has been validated in other conditions, such as sickle cell disease, ,,6,10,12,15 and the data presented in this study support exploring this approach in DS.

\section{Acknowledgments}

We would like to acknowledge the support of The Kids At Heart Neurosurgery Research Fund, The Justin Doo Moyamoya Research Foundation, and the Chae Family Fund.

\section{References}

1. Boggs S, Hariharan SL: An uncommon presentation of stroke in a child with trisomy 21. Pediatr Emerg Care 24:230-232, 2008

2. Dobson SR, Holden KR, Nietert PJ, Cure JK, Laver JH, Disco D, et al: Moyamoya syndrome in childhood sickle cell disease: a predictive factor for recurrent cerebrovascular events. Blood 99:3144-3150, 2002

3. Erguven M, Deveci M, Turgut T: Moyamoya disease and Down syndrome. Indian J Pediatr 72:697-699, 2005

4. Fukui M: Guidelines for the diagnosis and treatment of spontaneous occlusion of the circle of Willis ('moyamoya' disease). Research Committee on Spontaneous Occlusion of the Circle of Willis (Moyamoya Disease) of the Ministry of Health and Welfare, Japan. Clin Neurol Neurosurg 99 (Suppl 2):S238-S240, 1997

5. Golby AJ, Marks MP, Thompson RC, Steinberg GK: Direct and combined revascularization in pediatric moyamoya disease. Neurosurgery 45:50-60, 1999

6. Hankinson TC, Bohman LE, Heyer G, Licursi M, Ghatan S, Feldstein NA, et al: Surgical treatment of moyamoya syndrome in patients with sickle cell anemia: outcome following encephaloduroarteriosynangiosis. J Neurosurg Pediatr 1: 211-216, 2008
7. Jea A, Smith ER, Robertson R, Scott RM: Moyamoya syndrome associated with Down syndrome: outcome after surgical revascularization. Pediatrics 116:e694-e701, 2005

8. Junqueira PA, Moura-Ribeiro MV: [Moyamoya and Down syndrome: study conducted by meta-analysis.] Arq Neuropsiquiatr 60 (2-A):274-280, 2002 (Portuguese)

9. Kainth DS, Chaudhry SA, Kainth HS, Suri FK, Qureshi AI: Prevalence and characteristics of concurrent down syndrome in patients with moyamoya disease. Neurosurgery 72:210215,2013

10. Lin N, Baird L, Koss M, Kopecky KE, Gone E, Ullrich NJ, et al: Discovery of asymptomatic moyamoya arteriopathy in pediatric syndromic populations: radiographic and clinical progression. Neurosurg Focus 31(6):E6, 2011

11. Pysden K, Fallon P, Moorthy B, Ganesan V: Presumed perinatal stroke in a child with Down syndrome and moyamoya disease. Dev Med Child Neurol 52:212-214, 2010

12. Roach ES, Golomb MR, Adams R, Biller J, Daniels S, Deveber G, et al: Management of stroke in infants and children: a scientific statement from a Special Writing Group of the American Heart Association Stroke Council and the Council on Cardiovascular Disease in the Young. Stroke 39:26442691, 2008

13. Scott RM, Smith ER: Moyamoya disease and moyamoya syndrome. N Engl J Med 360:1226-1237, 2009

14. Scott RM, Smith JL, Robertson RL, Madsen JR, Soriano SG, Rockoff MA: Long-term outcome in children with moyamoya syndrome after cranial revascularization by pial synangiosis. J Neurosurg 100 (2 Suppl Pediatrics):142-149, 2004

15. Smith ER, McClain CD, Heeney M, Scott RM: Pial synangiosis in patients with moyamoya syndrome and sickle cell anemia: perioperative management and surgical outcome. Neurosurg Focus 26(4):E10, 2009

16. Smith ER, Scott RM: Spontaneous occlusion of the circle of Willis in children: pediatric moyamoya summary with proposed evidence-based practice guidelines. A review. J Neurosurg Pediatr 9(4):353-360, 2012

17. Sulter G, Steen C, De Keyser J: Use of the Barthel index and modified Rankin scale in acute stroke trials. Stroke 30:15381541, 1999

18. Suzuki J, Takaku A: Cerebrovascular "moyamoya" disease. Disease showing abnormal net-like vessels in base of brain. Arch Neurol 20:288-299, 1969

19. Veeravagu A, Guzman R, Patil CG, Hou LC, Lee M, Steinberg GK: Moyamoya disease in pediatric patients: outcomes of neurosurgical interventions. Neurosurg Focus 24(2):E16, 2008

20. Yamada I, Matsushima Y, Suzuki S: Childhood moyamoya disease before and after encephalo-duro-arterio-synangiosis: an angiographic study. Neuroradiology 34:318-322, 1992

\section{Author Contributions \\ Conception and design: Smith. Acquisition of data: Smith, See, Underberg. Analysis and interpretation of data: Smith, See, Rop- per, Robertson, Scott. Drafting the article: See, Ropper. Critically revising the article: Smith, Robertson, Scott. Reviewed submitted version of manuscript: Smith, Robertson, Scott. Approved the final version of the manuscript on behalf of all authors: Smith. Administrative/technical/material support: Underberg.}

\section{Correspondence}

Edward R. Smith, Department of Neurological Surgery, Boston Children's Hospital and Harvard Medical School, 300 Longwood Ave., Boston, MA 02115. email: edward.smith@childrens. harvard .edu. 